\title{
Fast Prototyping of the Internet of Things solutions with IBM Bluemix
}

\author{
Aleksey Popov and Andrey Proletarsky \\ Bauman Moscow State Technical University, \\ Moscow, Russia \\ alexpopov@bmstu.ru,pav@bmstu.ru
}

\author{
Sergey Belov and Alexander Sorokin \\ IBM Corporation, \\ Presnenskaya emb. 5, Moscow, Russia \\ Sergey_Belov@ru.ibm.com, alexander_sorokin@ru.ibm.com
}

\begin{abstract}
Fast prototyping for IoT projects has gained attraction in many industries. Today's IT market requires new faster techniques to get business advantages in different industries starting from the energy consumption and retail to the manufacturing, services, and agriculture. Combining sensors and actuators, embedded systems and networks with cloud computing platforms and cognitive services in one project is a very promising approach to address industry needs. Thus, system developers have to be familiar with many design technologies and best practices. At the same time, this approach requires a profound change in the way of interaction between major IoT market actors: suppliers and consumers of cloud platfrm and services, teams of developers and universities. In this paper, we analyze how to build effectively interaction of major IoT market actors and discuss a platform for such collaboration. We present a collaborative framework for fast prototyping of IoT solutions with different stakeholders participating. The paper demonstrates the results of this approach in the case of interaction between vendor, university, and industry. We consider a number of technological and practical aspects of this collaborative framework using IBM Bluemix cloud platform and IoT templates. We tested this approach in IoT hackathon with a participation of a vendor, local business partners, and industry representatives. Projects developed during this hackathon will be used to illustrate results achieved by applying introduced concept for IoT solutions prototyping.
\end{abstract}

\section{Introduction}

In order to achieve the sustainable development, humanity is now faced with global problems of air and soil contamination, lack of drinking water, carbon dioxide emissions, the fast growing city population and many others. Therefore we need the new powerful concept to improve and optimize our life everywhere where it is possible. The Internet of Things as the promising information technology gives the ability to understand and control physical processes much better than ever before. The true challenge today is to get maximum value from the Internet of Things and Smart technology incorporated into factories, cities, buildings, retail environment, agriculture and healthcare [1].

The IoT is usually defined as the technology to digitize and connect everything into computing networks [2]. Billions of physical assets already generating data volumes twice as fast as social networks and traditional computers [3]. Highly varied and noisy streaming data with short lifetime make it difficult to implement effective IoT projects in reasonable time. Hence the most IoT data are not used for an active analytic optimization and control, but for single event detection. To reduce the complexity and to release the potential of data analytic and Smart services, the information industry needs to implement robust cloud technology and cognitive computing.

The speed of development is expected to grow significantly, which requires jointly orchestrated efforts of all parties involved. The major resource to increase development speed is drastically reducing time to prototype the product while keeping its core analytic functionality. Fortunately with new cloud platforms for developers with a rich set of services embedded it now becomes possible to implement a prototype in just a few hours by applying the new approach, which will be given in later analysis.

With great progress made in recent years with scalable cloud computing, the development paradigm shifting from the computation time to the development speed optimization. The fast-changing market makes time of development the most valuable resource than any others. That means besides other things that the IoT education should be of more interest in academic world [4]. We also believe that universities can play an even more important role in the new technological environment by addressing new issues which all parties involved in IoT projects are facing, by testing new frameworks in student teams projects, hackathons, etc.

The paper is organized as follows. In section 2 we will consider the production chain for IoT projects and introduce general collaborative framework. In Section 3 


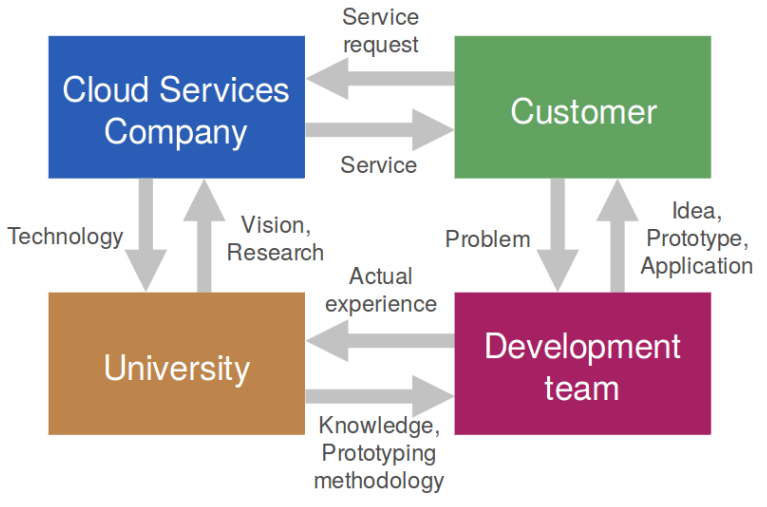

Figure 1. Internet of Things supply chain

we move on to build a practical template for fast prototyping of IoT services which will include infrastructure component. In section 4, we'll give the description of IoT hackathon at the Technical University. The projects completed during a hackathon organized jointly by IBM in Bauman Moscow State Technical University will be shown in section 5. A successful project started during this hackathon which was funded by a client right after, will be demonstrated in section 6 . In section 7 , we present the conclusions and discuss next steps.

\section{Problem Definition}

We define four actors in the IoT supply chain: a cloud service company (i); universities or academic technology centers (ii); creative development teams or individuals (iii); and customers (iv). Figure 1 shows the basic concept of an IoT supply chain interactions: the clockwise process of experience movement goes from cloud service company to customers (i); and the counterclockwise process of technology movement - from customers to cloud service company(ii). While this area is relatively new for the research, we discuss some related works.

The IoT supply chain can be defined as an intelligent interconnected network that binds together multiple tiers of suppliers, contract manufacturers, service providers, distributors and customers, physically located across different regions of the world [5]. By improving the entire supply chain efficiency all trading partners will greatly increase the economic benefit [6]. Taking into account the important role of universities and the sustainable technologies improvement we revise this approach with the IoT supply chain (as it depicted in Figure 1).

To make profitable business long term, major play- ers in cloud market should create an active ecosystem of development teams across the globe. Therefore, cloud enterprises have to collaborate with universities to provide practices to the market and to get an experienced feedback. We identify at least four challenging issues that can be effectively addressed here by the university research institutes.

The professional competencies problem: IoT industry will require an interdisciplinary education and a flexible stuffing. Students as the most driven and creative workers, have to be involved in the developing. With big ambitions and wide skills, student society generates most of the startups now. Therefore, IoT-enabled universities are possibly the best place to produce developers for the innovation industry.

The actual experience problem: This issue addressed to distribute recent experience and best practices inside the ecosystem. When new assets or services appear in the market, they should be tested and implemented to demonstrate best use cases and carry out improvements.

The technology availability problem: This issue can be specified because most of IoT cases are based on hardware and software. The technology availability assumes the open access to the developing facilities to enable the cost and time effective prototyping. Solved by the universities technology centres, it allows to perform the advanced technology delivering into the developer's society, helps to accumulate best experiences, advances the methodological component of education. We would note, that this approach is relevant and is being implemented more or less.

The fundamental research problem: This issue is based on the market opportunities rather than pure functional value, because of all IT industry focused on the demand generation. Therefore, the sufficient time and analysis are required to summarize the actual market experiences and combine them with technology factors.

The challenge of bringing hundreds of teams of highly professional IoT developers for sustainable development is difficult to address. Universities turn out to be ideally located on IoT development crossroads, so both industry and development team can leverage it to start a number of small projects when a large number of multidisciplinary developers required.

To understand the variety of skills for the IoT development let's consider a situation, where we analyze sensor data and control actuators by making decisions on the cloud platform. The most common educational example is the irrigational systems in the greenhouse [7, 8] or trash can in the Smart city [9]. First, developers need to work with sensors on a physical layer that require at least the hardware developer who will be 
able to solve the electrical connectivity problems and to register analog or digital information. Then they need to implement the system on chip (SoC) to manipulate primary data and share them with other things. The next step requires understanding networking principles to connect multiple SoC into interconnected network [10]. IoT devices should communicate with each other and with cloud platform through such protocols like HTTP or MQTT [11]. This requires SoC software developer familiar with programming in various operational systems (primary on Linux and Microsoft Windows 10) [12] and programming languages (most useful are: $\mathrm{C} / \mathrm{C}++$, Python, JavaScript, Java, Ruby).

Cloud technology is another significant piece of knowledge for IoT projects. Developing on the cloud platform is dealing with the target problem and defining the system architecture. It requires a substantial experience to use the appropriate cloud services to rightfully address project needs. That could be difficult even for skilled developers when they have to process a large amount of data. Then the project should be supported with the web and mobile developer who is able to provide data visualization. If the project needs the data analyzing, it is necessary to have skills in statistics to implement an analytical technology. Finally, the team should be led by experienced and innovative thinking manager.

The natural solution we see here is to create a set of IoT templates which can be used by developers for fast prototyping. In a market with a great number of IoT applications, the cloud services company will actually face the problem of a technology distribution. The skilled developer could take a role to implement technology basics and to support the end user locally. Such a set of concept solutions can be built in collaboration with universities and have to cover most of IoT implementations. We show one of such template in the next section.

\section{IoT template solution}

The R\&D time for IoT projects is critical for survive. In many cases, the innovative project is able to win the on-premises system when it has the key advantage with a faster path to market. Thus it is significant to implement the set of improved instruments for robust prototyping and flexible services for developer needs [13]. The best practice is to implement the prebuild "starter kit" including cloud-based platform, web hosting resources, System-on-Chip (SoC) microcomputers with simplified and reliable interfaces, common usable sensors and actuators. In this work, we describe such a kit based on IBM Bluemix with pre-installed analytic services (IBM Watson IoT, Cloudant, dashDB, R-Studio, and Node.js) and device IoT layer (RaspberryPi, Arduino, Grove sensors and wireless interfaces). The advantages of this platform with examples we got during the student hackathon in Bauman Moscow State Technical University are shown below. As a result, 36 hours of intensive development and $150 \$$ per kit was enough for every team to build working and very exciting IoT showcase.

Since the cloud has become the primary platform to host IoT services [14], developers need to integrate cloud-based resources into the project architecture. [15] shown the key technologies to implement an IoT solution in agriculture and discovered the template which is based on ZigBee networks. This approach may be extended with analytical services and cloud-based infrastructure as well. [16] attempted to categorize the services provided by the IoT in order to help application developers build upon a base service. They outlined four main categories of IoT services and provided some useful examples: Identity-Related Services (i); Information Aggregation Services (ii); Collaborative-Aware Services (iii); Ubiquitous Services (iv). Based on this research we define here the "starting kit" of services combined with solution network to attract the fast prototyping, and provide the IoT template solution with appropriate methodical maintenance.

The target cloud platform has to be enabled for the Internet of Things: it should be scalable to be connected with many thing instances; flexible to implement new features and services; and simple to reduce the development time. There is a list of candidates, such as Microsoft Azure, Google Cloud platform, and others. In this work, we describe IBM Bluemix (Platform as a Service, PaaS) cloud to implement IoT template solution.

Bluemix is the cloud platform for the rapid prototyping of various services and applications including mobile and web kits, databases, programming environments and SDK, data analytics tools, Internet of Things and cognitive computing infrastructure. It is based on open source computer technologies like Cloud Foundry and Docker $[17,18]$. To simplify the monitoring and control functions, Bluemix provides the access through the dashboard and Cloud Foundry console [19]. It should be noticed that in many cases IBM provides easy access to faculties, students, and developers to Bluemix.

To support students with IoT concept, we developed the basic cloud-based system for a wide area of implementations. An illustration of the introduced concepts is depicted in Figure 2. The system carries out the list of functionality:

- Hosts at the bottom of hierarchy interact with multiple physical objects. Through sensors and actuators, the IoT system registers current states of con- 


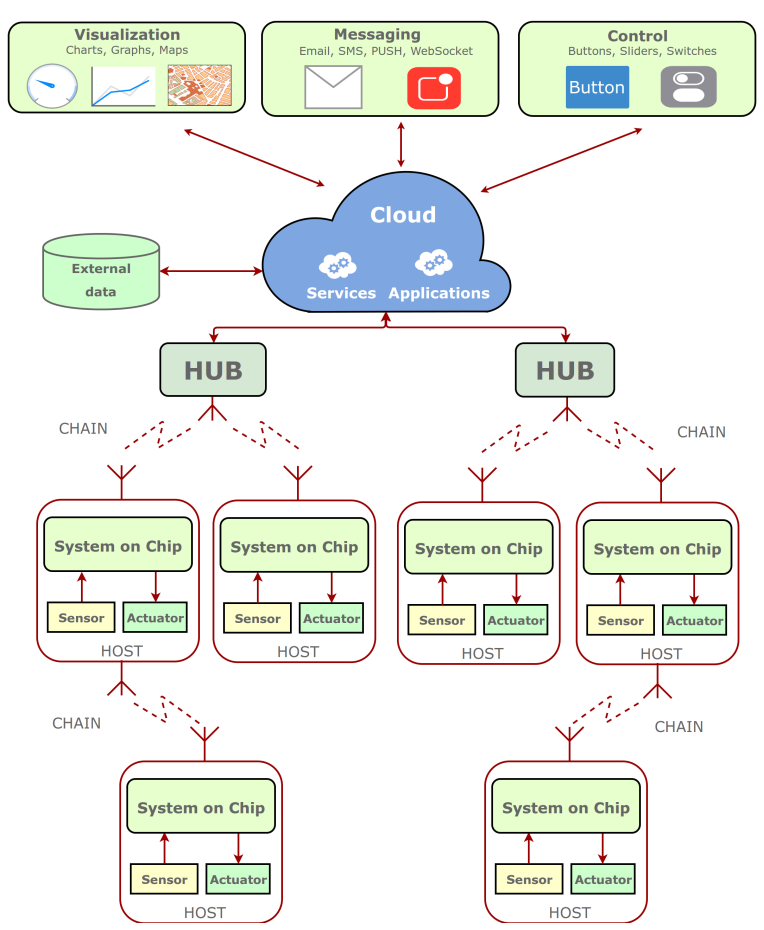

Figure 2. Internet of Things template solution

trollable things and able to change them.

- SoC inside the host receives the primary data from sensors for their further filtering and uploading. It allows defining on this level the appropriate period for data uploading depending on the physical process speed.

- Multiple hosts can be distributed on a large area with physical objects. To cover more area and improve functionality they could be interconnected through another host into the chain [20].

- Data is sent to a hub or another host by the wireless interface like RF link, ZigBee, Bluetooth, WiFi, 6LoWPAN[21]. The appropriate interface can be defined by comparing such parameters as bitrates, encryption details, power consumption options, signal coverage area and other features.

- The primary data are processing in a hub to form messages for the cloud platform. This is usually understanding as packing data into the JSON object and sending it to the message queue over TCP/IP stack. Hub is usually wirable connected to the Internet and represents multiple single hosts.

- The cloud platform receives data from multiple sources to store, analyze, and visualize them.

- Custom applications on the PaaS cloud are using the predefined cloud services to access stored data. For example, the application can receive HTTP re- quest, parse and store it with the database service. Other application can get data from the database and generate the web page to visualize statistics with graphs, charts, bevels, bars etc. The cloud application can send messages to the end user by SMS or push messaging.

- External data can be used by applications to improve analytic functions. For example, an application is able to predict KPI's values on historical data.

- The IoT project can use HTML pages to visualize things states and KPIs.

The template solution shown in Figure 2 can be extended with more than 100 prebuild services from a Bluemix catalog to support mobile development, version control, web applications, integration control, networking, and APIs. In addition, Bluemix allows to build a new service from the Docker container and attach it to the project through WebSockets or HTTP connections. This basic concept can be implemented in many areas to build IoT use cases. For example, it can be used to track the health data on a host device and move them through the mobile network or wifi to the cloud services. Another way, for Smart homes this concept can be used as the energy efficiency improvement, home security, and environment control. As for Smart Cities, it can improve the public infrastructure with traffic management, air and water quality monitoring or smart offices. Other powerful areas are agriculture and smart manufacturing.

\section{Hackathon for IoT prototyping}

A hackathon for the hardware and software development differs with that one just for programmers. It needs from organizers to be well equipped with embedded systems both widely skilled in programming and cloud infrastructure. To be successful, organizers should plan the preferred set of hardware and support teams with the appropriate environment. Unlike single programming, the hardware and software development needs individual kits for every team (sensors, systems on a chip, wireless interfaces, debug tools) and commonly used additional tools (oscilloscopes, power supplies, digital analyzers, soldering stations and other useful equipment). As our practice and Murphy's law has shown, all of the additional equipment was useful to get the best result. For that purpose, we used development resources from the Bauman MSTU Internet of Things laboratory.

Depending on the features, the price for additional equipment starts from $1000 \$$ and does not have any limits from above. Hopefully, we used generic parts with low frequency and our additional expenses were near the $1500 \$$. As for the individual set, we spent about $2000 \$$ 


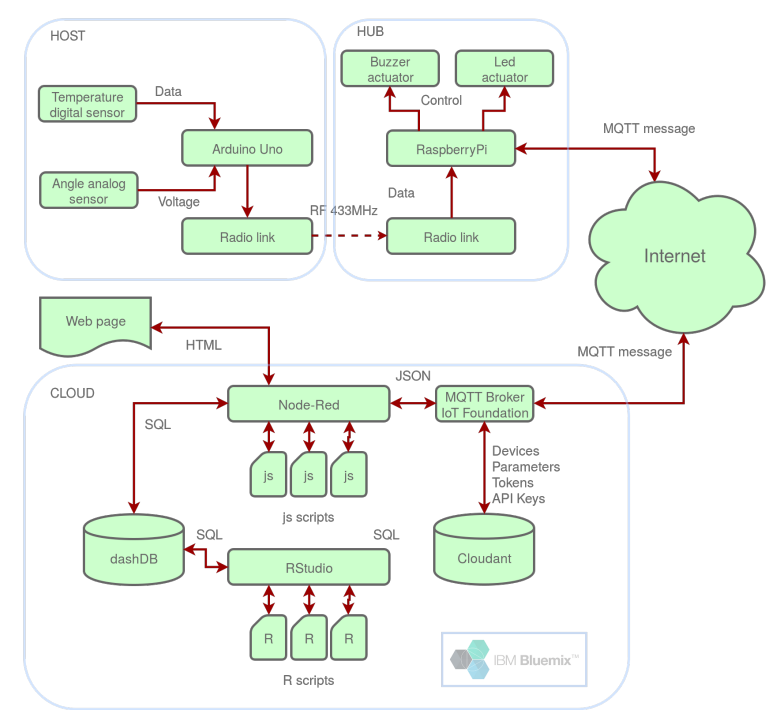

Figure 3. Internet of Things fast prototyping project's architecture

per 14 teams, therefore $150 \$$ is not so expensive for one IoT showcase.

Let us get back to the concept. To be implemented in multiple instances, the concept solution infrastructure was actualized into the educational project with a kit of useful and inexpensive environment [22]:

- LEDs and buzzers were used as simple actuators.

- More than a hundred of sensors was prepared for projects development, but just the analog angle sensors and the digital temperature sensors were actually used in the educational project. We preferably used the simplified Grove interface to avoid short circuits.

- Arduino Uno microcomputers with Grove extension board used to receive data from sensors.

- Wireless radio frequency modules RF $433 \mathrm{MHz}$ used to switch host with a hub.

- RaspberryPi microcomputer used as a computing hub and to control actuators. Through the RF link and Ethernet, it can be connected to a host and to the cloud side.

- IBM Bluemix implemented data storage, analytical processing, and data visualization services.

The prototyping project's architecture is shown in Figure 3. The project works as follows. Arduino registers analog and digital data from sensors and sends them through the radio link. Such a link allows to data transmit from multiple hosts to the one or more computing hubs (RaspberryPi). The hub forms MQTT packets and transmits them to the Bluemix cloud for storage and processing. Back to the hub, Bluemix sends control com- mands to actuators.

Let describe cloud part of the project in more details. MQTT packets are moving to the message broker inside the Watson IoT service (also called IoT Foundation). It stores message queue and connection details in the attached Cloudant database. The main application is based on Node.js framework and could be written with Node-Red visual editor inside the Bluemix. This tool is very friendly for beginners and positioned as one of the most useful Internet of Things online SDK. Node.js code primitives (nodes on Node-Red) are connected through multiple data streams into flows. The first flow in our project receives the data stream from IoT Foundation and stores it into the dashDB database. Another flow runs the predefined script in $\mathrm{R}$ language to perform a regression analysis of stored data. This $\mathrm{R}$ script determines predictive values for some of the upcoming moments and sends them back to the dashDB and Node.js application.

According to the obtained primary data and analysis results, the application flow builds the web page with charts and control buttons. A client can view charts in a browser and produce operations by clicking the control button. After this, the application flow in Node-Red sends the MQTT command through the IoT Foundation service back to the hub. This takes an action to the LED and buzzer attached with it. In the case when the predictive value exceeds the limit, an application flow automatically sends the MQTT command to switch the buzzer on.

The project was divided into four stages:

- Stage 1. Build and verify host with sensors

- Stage 2. Build hub and receive data from the host

- Stage 3. Make Bluemix template solutions and build visualization on Node-Red

- Stage 4. Make predictive analysis and send commands back to actuators

As we noticed, this requires up to 6 hours to be build. After that, all hackathon teams started to make their innovative projects.

\section{Projects description}

Here is a short summary of some projects done by students during our hackathon. In the next section we will describe one commercially successful project.

\subsection{Smart crossroad}

The crossroad control system analyses data from multiple sources to focus appropriate attention on citizens movement. At first, it uses the cars traffic from the GIS portal (maps.yandex.ru) and people traffic from city 
events system (afisha.yandex.ru). Those data getting to the Bluemix cloud to predict the citizens and cars traffic near the crossroad. Next, the system sensing the around area to understand citizen positions and light intensity. All this information used by Smart crossroad to understand how dangerous the crossroad is at the moment. As a result, system controls smart backlights to make attention to citizens for car drivers to make city crossroads more safety. In addition, video camera, attached to the system, captures vehicles near the stop line.

\subsection{NRget(x)}

This system allows distributing energy flows from many sources to save energy for smart homes and smart cities. For that purpose, it analyses the data from external sources and the information from solar batteries, wind generators, accumulators to control the stored energy. System defines:

- how much energy is produced from power generators?

- what is the required power at the present moment?

- how much energy was stored before?

This allows defining how much energy should be stored, sold or bought for effective energy consumption.

\subsection{Smart Pill Box}

This project is for those people who have difficulties while taking medicine. The problem is that many people often miss the appointed time because of poor memory. People with disabilities such as bad eyesight sometimes mix pills up. The team created a smart pill box which announces users when they should take their medicine and lights up the required compartment. The hardware consists of the pill box with sensors, LEDs, and buttons, while the software is based on the Bluemix cloud services and Android application.

\subsection{RealClimate}

This project designed to capture and replicate various climate conditions. The idea is to create the climate conditions in real time and equal to that one in another place. The project consists of 3 different modules. The first module is meant to be placed in natural conditions, collect data about the surrounding climate (temperature, humidity, soil moisture, etc.), and upload the collected data to the Bluemix. The second module is placed in the area, where the desired climate is to be replicated. It collects information about the second climate and uploads it to the cloud. The third module is connected to various systems for recreating climate conditions (AC, irrigation, humidifier, etc.).
Information in the cloud from the two climate zones is compared, and commands are sent to the third module to enable/disable the necessary subsystems. This project has many potential use cases, four of which are listed below:

- Zoos and arboretums: with the help of this product, stress associated with transporting plants and animals can be reduced to a minimum. The opposite effect can also be used for creating natural stress, which strengthens the immune system and increases vitality. This can be achieved by monitoring and recreating climate conditions in real-time.

- Industrial use: in farms and greenhouses for increasing crop yield.

- Virtual reality systems: for creating a more immersive experience.

- Science: for tracking and analyzing climate changes on planet Earth (and other planets in the future). This can also help forecast climate anomalies.

\subsection{Smart Escalator}

The target of the Smart Escalator project is to distribute traffic flow between underground escalators according to accident prevention rules. Expected traffic flow calculation is based on readings of sensors built in turnstiles and also on analyzed statistics. Statistical data is being collected during previous several weeks so there's ability to predict future traffic flow in the certain hour of the certain day (traffic difference between weekdays and holidays is also considered).

Parsing of Internet resources allows to precise prediction according to the future or current events near certain subway station (e.g. public events like sports matches, nearby stations stoppage and so on). The smart system makes a decision to turn off and on escalators and reversing their direction. When they are stopped or reversed the system informs passengers with indicators, closes the entrance to the escalator and completes one full turning cycle of stairs. Then the system closes the escalator and performs operations for stopping or changing direction. With this feature, the project increases the safety and convenience level of subway transportation.

\subsection{M\&F}

This project is to identify people by using IBM Watson visual recognition service. The problem is to find the moment when face position is most useful for capturing and recognition. For this purpose, the ultrasonic range finder detects an object at a correct distance from the camera. Therefore it does not allow to capture the random objects outside the recognition zone. After cap- 


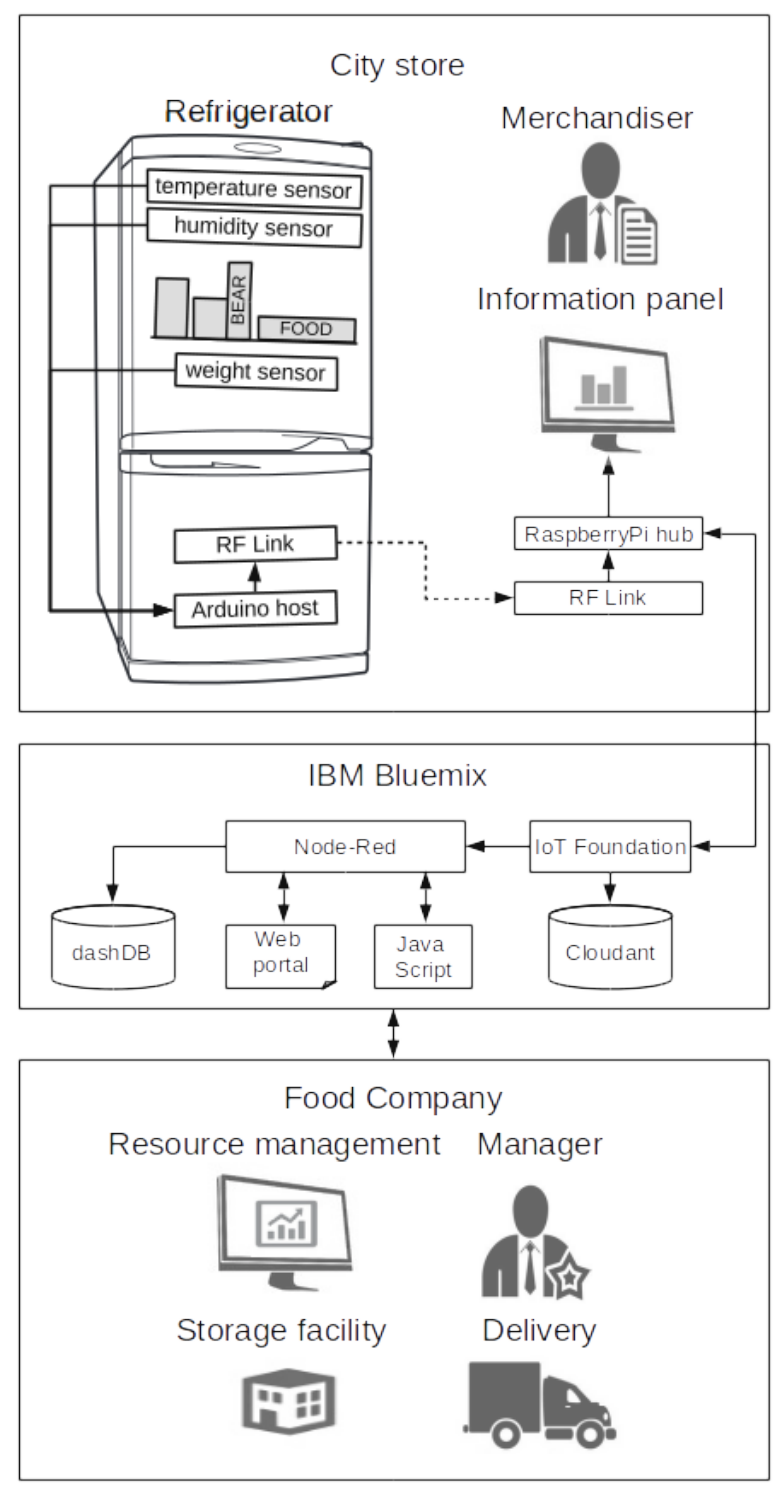

Figure 4. Smart refrigerator project

turing, the picture is transmitting to the Bluemix for further recognition. Thus, the image is comparing with predefined faces on Watson service. If the person is successfully identified, Node.js application sending MQTT message to the actuator (lock subsystem, alarm etc.). This project results can be used for security in Smart cities, Smart homes and Smart offices.

\section{Example of commercial project}

The commercial success of the Internet of Things project is the result of the effective solution with a small cost. Such a project was immediately demanded by the business after the hackathon. The Smart refrigerator system aimed to upgrade refrigerators with functions to get information about products being stored and to send data to the cloud analyzing the system. This cost-effective system is based on Arduino, RaspberryPi, and Bluemix to define the amount of stored food on every refrigerator's shelf. If the weight is lower than normal limit, the system automatically sends an order to the online store. In addition, the system monitors food terms and sends alerts to the LCD and web panels (for example, when food was stored on the shelf for a long time).

Inside the refrigerator, the weight sensor, temperature and humidity sensors are used to define store conditions. All the data sent by the Arduino are following to the RaspberryPi computing hub. This device visualizes the food weight and store conditions for local user and sends it to the cloud. IBM Bluemix is used to analyze and visualize data from multiple shelves and refrigerators. Node.js application stores data in the dashDB database makes orders for food restocking and visualize analysis results.

This idea of a refrigerator which able to sense the kind of products is not new [23]. But the existing smart refrigerators are expensive and require additional support. As a result, this project got the attention from the food market companies. The brewer company thinking about how to control the beer supplies on the refrigerators shelves in city stores. Existing equipment does not allow to determine the storage conditions and inventory. Therefore, it is necessary to develop a system to get information from many refrigerators around the city and analyze it to schedule automatic delivery. The company can ask merchandiser in every store to distribute beer inventories on the shelves or refrigerators (Figure 4) if needed. The system automatically checks the storage conditions and sends information to the beer company and to the merchandiser. If those conditions are violated, this automatically raises the request to support service. The brewer company aims to analyze data to create the best conditions for sales. Smart refrigerator system can help company to understand:

- how many beer bottles sold in different stores.

- how to distribute bottles on the refrigerator shelves in the best way.

- how many beer bottles should be delivered to the store?

- the beer storage conditions.

\section{Conclusion}

We introduced the fast prototyping technique for the Internet of Things projects to make them competitive 
enough to be already acquired by business. The shown IoT template solution is based on inexpensive components and the IBM Bluemix cloud platform. The concept was implemented as the educational project to be helpful piloted at the IoT hackathon in Bauman Moscow State Technical University. We shown some innovative projects developed by the hackathon teams.

Smart refrigerator project allows retail companies to analyze information about the products in city stores. The brewer company used this project to create an analytical system for schedule automatic delivery and check storage conditions.

More detailed framework to address fully industry need is yet to be developed and it could be a good area for further research. We also plan to create a network of IoT labs in technical universities in close collaboration with industry. It can extend both the set of IoT template solutions and perspective showcases.

\section{References}

[1] S. Haller, S. Karnouskos, and C. Schroth, "The Internet of Things in an Enterprise Context, FIS 2008, Vienna, Austria, September 29-30, 2008, Springer Berlin Heidelberg, 2009, pp.14-28.

[2] H. R. Schindler, J. Cave, N. Robinson, V. Horvath, P.J. Hackett, S. Gunashekar, M. Botterman, S. Forge, H. Graux, European's policy options for a dynamic and trustworthy development of the Internet of Things. RAND Europe, 2013, p. 221.

[3] J. Manyika, M. Chui, P. Bisson, J. Woetzel, R. Dobbs, J. Bughin, D. Aharon, The Internet of Things: Mapping the value beyond the hype, McKinsey Global Institute, June 1, 2015, p. 24.

[4] G. Kortuem, A. K. Bandara, N. Smith, M. Richards, M. Petre, "Educating the Internet-of-Things Generation", IEEE Computer, Feb. 2013, vol. 46 no. 2, pp. 53-61.

[5] L. Wadhwa, "How the IoT will Change the Supply Chain?", AVNet, 2016, [Online]. Available: http://app.em.avnet.info/e/ er?s=1224369867\&lid=1241

[6] Y. Gu and T. Jing, "The IOT research in supply chain management of fresh agricultural products," Artificial Intelligence, Management Science and Electronic Commerce (AIMSEC), 2011 2nd International Conference on, Deng Leng, 2011, pp. 7382-7385.

[7] N.S. Viswanath, S. P. Bai, P. Rajesh, N.B. Mallikarjuna, "IOT based green house monitoring system", International Journal of Electronics and Communication, Engineering and Technology (IJECET), Volume 6, Issue 6, June, 2015, pp. 45-47.
[8] Ajeetkumar Rai, Sarfarajahamadidrisi and Shahbaz Ahmad, "An Experimental Study of Forced Convection Green House Drying", International Journal of Advanced Research in Engineering and Technology (IJARET), Volume 4, Issue 5, 2013, pp. 10-16.

[9] S. Phithakkitnukoon, M. I. Wolf, D. Offenhuber, D. Lee, A. Biderman and C. Ratti, "Tracking Trash," in IEEE Pervasive Computing, vol. 12, no. 2, AprilJune 2013, pp. 38-48.

[10] N. Matthys et al.., "uPnP-Mesh: The plug-andplay mesh network for the Internet of Things," Internet of Things (WF-IoT), 2015 IEEE 2nd World Forum, Milan, 2015, pp. 311-315.

[11] B. Boyd, J. Gauci, M. P Robertson, N. Van Duy, R. Gupta, V. Gucer, V. Kislicins, Building Realtime Mobile Solutions with MQTT and IBM MessageSight, IBM Redbook, SG24-8228-00, October 2014. p. 268.

[12] A.S. Tanenbaum, and H. Bos, Modern Operating Systems: Global Edition, Pearson Education Limited, 2015. p. 1137.

[13] A. Fisher, "Best practices for IoT development. Applying lessons from mobile development to your IoT projects". IBM Corporation. 2015. [Online]. Available: http://www.ibm.com/developerworks/library/iotmobile-practices-iot-success/

[14] A. Botta, W. de Donato, V. Persico and A. Pescap, "On the Integration of Cloud Computing and Internet of Things," Future Internet of Things and Cloud (FiCloud), 2014 International Conference, Barcelona, 2014, pp. 23-30.

[15] X.-Y. Chen, and Z.-G. Jin, "Research on Key Technology and Applications for Internet of Things," 2012 International Conference on Medical Physics and Biomedical Engineering, Physics Procedia, 33, 2012. pp. 561-566.

[16] M. Gigli, and S. Koo, "Internet of Things, Services and Applications Categorization," Advances in Internet of Things, 1, 2011. pp. 27-31.

[17] M. Kim et al., "Building scalable, secure, multitenant cloud services on IBM Bluemix," in IBM Journal of Research and Development, vol. 60, no. 2-3, March-May 2016. pp. 8:1-8:12.

[18] B. Arnold et al., "Building the IBM Containers cloud service," in IBM Journal of Research and Development, vol. 60, no. 2-3, March-May 2016. pp. 9:1-9:12.

[19] A. Gheith et al., "IBM Bluemix Mobile Cloud Services," in IBM Journal of Research and Development, vol. 60, no. 2-3, March-May 2016. pp. 7:17:12.

[20] Device democracy. Saving the future of the Inter- 
net of Things / IBM Global Business Services Executive Report. IBM Institute for Business Value, GBE03620USEN, IBM Corporation, September 2014, p.28.

[21] Wireless connectivity for IoT applications. STMicroelectronics - October 2015. [Online]. Available: https://my.st.com/public/STe2ecommunities/ interface/default.aspx

[22] Open student hackathon for IoT solutions prototyping [Online]. Available: http://bmstuhackathon.github.io/example/

[23] Internet refrigerator [Online]. Available: https://en.wikipedia.org/wiki/Internet_refrigerator 